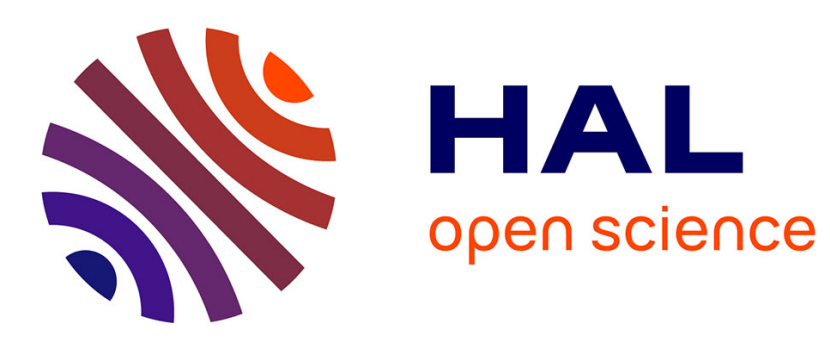

\title{
Stability and fluctuations of a spatially periodic convective flow
}

\author{
Y. Pomeau, P. Manneville
}

\section{To cite this version:}

Y. Pomeau, P. Manneville. Stability and fluctuations of a spatially periodic convective flow. Journal de Physique Lettres, 1979, 40 (23), pp.609-612. 10.1051/jphyslet:019790040023060900 . jpa-00231699

\section{HAL Id: jpa-00231699 https://hal.science/jpa-00231699}

Submitted on 1 Jan 1979

HAL is a multi-disciplinary open access archive for the deposit and dissemination of scientific research documents, whether they are published or not. The documents may come from teaching and research institutions in France or abroad, or from public or private research centers.
L'archive ouverte pluridisciplinaire HAL, est destinée au dépôt et à la diffusion de documents scientifiques de niveau recherche, publiés ou non, émanant des établissements d'enseignement et de recherche français ou étrangers, des laboratoires publics ou privés. 


\title{
LE JOURNAL DE PHYSIQUE-LETTRES
}

\section{Stability and fluctuations of a spatially periodic convective flow}

\author{
Y. Pomeau \\ DPh.T., CEN-SACLAY, B.P. nº 2, 91190 Gif-sur-Yvette, France \\ and P. Manneville \\ DPh.G.PSRM, CEN-SACLAY, B.P. nº 2, 91190 Gif-sur-Yvette, France
}

(Reçu le 27 août 1979, révisé le 2 octobre 1979, accepté le 16 octobre 1979)

\begin{abstract}
Résumé. - Nous analysons le seuil de convection d'une instabilité en rouleaux à l'aide d'une équation modèle. L'accroissement de la longueur d'onde est la conséquence d'un principe variationnel. Pour certaines longueurs d'onde, la diffusion de phase est instable vis-à-vis d'une compression (ou d'une dilatation) ou d'une torsion du système de rouleaux. Les fluctuations thermiques créent un mouvement brownien de translation de la structure qui pourrait être observable dans de bonnes conditions expérimentales, comme celle de l'instabilité de RayleighBénard dans l'hélium liquide ou Carr-Helfrich dans un cristal liquide nématique.
\end{abstract}

\begin{abstract}
Using a model equation we analyze the onset of convection for a roll instability. The wavelength increase follows from a variational approach. For certain wavelengths, phase diffusion turns out to be unstable against compression (or dilatation) and torsion of the rolls. Thermal phase fluctuations induce a tiny brownian motion of the structure which should be observable in carefully designed experiments such as Rayleigh-Bénard in liquid helium or Carr-Helfrich in nematic liquid crystals.
\end{abstract}

There are numerous examples of periodic spatial structures which occur in a system through a supercritical bifurcation and the Rayleigh-Bénard (R. B.) thermoconvective instability [1] is perhaps the most famous one. In this paper we discuss some properties of the non-linear regime close to the instability threshold. For convenience we use the terminology of thermoconvective instabilities but our discussion has a more general character. We use a description of the bifurcation from the conductive state to the convective state in terms of a simple but plausible model equation which allows for a twodimensional spatial dependence of the structure which we think to be more universal than the dependence on the third dimension closely connected to the details of the hydrodynamic problem. It will be shown that :

i) our model describes at least qualitatively the wavelength increase of the periodic structure in the vicinity of the instability threshold [2].

ii) For a system of parallel rolls, the dynamics of the phase variable - which describes a local translation of the rolls - is governed by two diffusion coefficients $D_{\|}$and $D_{\perp}$ which depend on the wave- vector of the underlying structure and on the distance to the threshold. Equations $D_{\|}=0$ and $D_{\perp}=0$ give the marginal stability condition for that structure against the two seemingly possible types of perturbation close to the threshold.

iii) Due to thermal noise [3] the phase fluctuates, which leads to a translational brownian motion of the structure. The corresponding diffusion coefficient will be evaluated and observational possibilities will be discussed.

1. The model equation and its stationary solution. The dynamics of a supercritical stationary bifurcation obey the Landau type equation [4] :

$$
\delta_{\mathrm{t}} A=\varepsilon A-A^{3}
$$

where $\varepsilon$ depends on the constraint applied to the system (the vertical temperature gradient in the R.B. case) and measures the distance to the threshold (negative below and positive above). $A$ is the amplitude of the convective state, the unstable Fourier component of the hydrodynamic velocity in the R.B. case. Owing to the symmetry $(A) \rightarrow(-A)$ no term in $A^{2}$ is present in eq. (1). 
To describe the spatial structure of the unstable mode, one must turn to an equation more complicated than (1) but still simpler than the full set of hydrodynamic equations. As indicated above, the single quantity $A$ now depends on two spatial coordinates. The required equation has to display translational and rotational invariance and must lead to a convective solution with a well defined wave-vector $q_{0}$ at threshold. In dimensionless notations the simplest candidate reads [5]

$$
\delta_{\mathrm{t}} A=\varepsilon A-\left(\Delta+q_{0}^{2}\right)^{2} A-A^{3}
$$

where $\Delta=\partial_{x x}^{2}+\partial_{y y}^{2}[6]$.

Eq. (2) defines a functional gradient flow. Indeed it may be written as $\partial_{\mathrm{t}} A=-\delta V[A] / \delta A$ where the potential $V$ reads :

$$
\begin{aligned}
V[A] & =\iint \mathrm{d} x \mathrm{~d} y \times \\
& \times\left\{\frac{1}{2}\left(q_{0}^{4}-\varepsilon\right) A^{2}+\frac{1}{4} A^{4}+\frac{1}{2}(\Delta A)^{2}-q_{0}^{2}(\nabla A)^{2}\right\} .
\end{aligned}
$$

When $\varepsilon$ is positive, the null solution is linearly unstable against a certain class of perturbations. Close to the threshold, these unstable perturbations have a wave-vector $q=q_{0}+\delta$ with $|\delta|<\delta_{\|}(\varepsilon)$ and

$$
\delta_{\|}(\varepsilon)=\left(\varepsilon / 4 q_{0}^{2}\right)^{1 / 2} \text {. }
$$

The unidimensional stationary periodic solution is expanded as

$$
A_{0}(x)=A^{(0)} \sin q x+A^{(1)} \sin 3 q x+\cdots
$$

where

$$
A^{(0)}=\left[\frac{4}{3}\left(\varepsilon-4 \delta^{2} q_{0}^{2}\right)\right]^{1 / 2}
$$

and

$$
A^{(1)}=\left(A^{(0)}\right)^{3} / 256 q_{0}^{4} .
$$

Minimization of $V[A]$ with respect to $q$ leads to :

$$
\delta / q_{0}=-\varepsilon^{2} / 1024 q_{0}^{8}
$$

i.e. to a slight increase of the wavelength. This is the result of the contribution to $V[A]$ of the harmonics generated by the non-linearities. In $V[A]$, the term $(\Delta A)^{2}$ encourages small wave-vectors while $-(\nabla A)^{2}$ favours large ones, but the contribution of harmonics increases the weight of the first term $\left(\sim q^{4}\right)$ more rapidly than the second one $\left(\sim q^{2}\right)$ so that the optimum is found at $q<q_{0}$.

This optimum cannot be reached through instabilities of a periodic infinite pattern with an imposed wave-number since as small as $\delta$ be, one cannot go from $\sin (k x)$ to $\sin ((k+\delta) x)$ by infinitesimal local (i.e. in $x$ ) changes. The wavelength adjustment is supported by numerical simulation on eq. (2) in finite geometry and turns out to be in qualitative agreement with experimental observations [2]. It is made possible (both in computer experiments and in reality) by the disappearance of rolls near the boundaries (or at the center in cylindrical geometry). It is possible that for very peculiar choice of initial conditions the absolute minimum of $V$ compatible with the boundary conditions is not reached. However in a large (1-dimensional) box, the optimization of $V[A]$ is governed by the bulk contribution, proportional to the size of the system, so that the wavelength is determined by the optimum of $V[A]$ as computed for an infinite pattern, neglecting the boundary layer contribution.

2. Phase diffusion process. - Owing to translational invariance, if $A_{0}(x)$ is a solution, then another solution is $A_{0}(x+\varphi)$ where $\varphi$ is an arbitrary constant phase. If $\varphi$ is slowly varying in space and time, $A_{0}[x+\varphi(x, y, t)]$ is close to being a solution. Assuming $\varphi$ to be small one looks for the solution of eq. (2) under the form :

$$
A(x, y, t)=A_{0}(x)+\varphi(x, y, t) \partial_{x} A_{0}+A_{1}+A_{2}+\text { etc... }
$$

where the expansion parameter is $\nabla$ and $A_{1} \sim \mathcal{O}(\nabla \varphi)$, $A_{2} \sim \mathcal{O}(\nabla \nabla \varphi)$, etc... If one looks at a stationary solution in this form, one solves at each order equations of the general form:

$$
\Lambda_{0} A_{n}(x)=b_{n}(x)\left(\nabla^{n} \varphi\right)
$$

where $\Lambda_{0}=\varepsilon-\left(\Delta+q_{0}^{2}\right)^{2}-3 A_{0}^{2}, b_{n}$ is known and $A_{n}$ unknown. Considering $\nabla^{n} \varphi$ as $x$-independent, since it varies slowly on the length scale $q_{0}^{-1}$, one can look for $A_{n}(x)$ under the form

$$
A_{n}(x)=\left(\nabla^{n} \varphi\right) \cdot a_{n}(x)
$$

which leads to

$$
\Lambda_{0} a_{n}(x)=b_{n}(x) .
$$

This equation has no solution in general, since 0 is an eigenvalue of $\Lambda_{0}$ with the eigenfunction $\partial_{x} A_{0}$, as it can be checked by derivation with respect to $x$ of eq. (2) (with $\partial_{t} \equiv 0$ ).

However (6) has a solution (up to arbitrary addition of a function proportional $\partial_{x} A_{0}$, that amounts to the arbitrary phase choice for $A_{0}$ ) if $b(x)$ has no component in the kernel of $\Lambda_{0}$. If this is the case, one says that the solvability condition of (6) is fulfilled. If, on the contrary this solvability condition is not fulfilled, one uses the apparent freedom in the choice of the time derivative $\varphi$ to satisfy the solubility condition. This derivation of an equation of motion for the slow mode parallels the Chapman-Enskog theory of transport phenomena in gases [7]. The underlying conserved quantity here is the number of rolls. This helps to understand that the existence 
of a slow diffusive motion is a very general feature of dissipative structure with an arbitrary phase choice, and is, of course not limited to this model problem. At order zero in $\nabla$, the linearized equation for $\varphi$ merely expresses the marginal stability of the structure against an uniform infinitesimal translation. It reads $\varphi \Lambda_{0} \partial_{x} A_{0}=0$. At first order in $\nabla$, with $A_{1}=a_{1}(x) \varphi_{x}$ one gets :

$$
\Lambda_{0} a_{1}=4\left(q_{0}^{2}+\partial_{x x}^{2}\right) \partial_{x x}^{2} A_{0} .
$$

The solubility condition for this equation is fulfilled since the r.h.s. is orthogonal (in the sense of the scalar product

$$
(f, g)=\int_{x}^{x+\lambda} f\left(x^{\prime}\right) g\left(x^{\prime}\right) \mathrm{d} x^{\prime}
$$

where $\lambda$ is the common period of $f$ and $g$ ) to the kernel $\partial_{x} A_{0}$ of the self-adjoint operator $\Lambda_{0}$. The second order problem looks similar eq. (7) but the solubility condition is no longer trivially fulfilled. It may be written as diffusion equations for $\varphi$ [8] :

$$
\partial_{t} \varphi=D_{\|} \partial_{x x}^{2} \varphi+D_{\perp} \partial_{y y}^{2} \varphi
$$

with

$$
\begin{aligned}
D_{\|}=-2\left(\partial_{x} A_{0},\left(q_{0}^{2}+\right.\right. & \left.3 \partial_{x x}^{2}\right) \partial_{x} A_{0}+ \\
& \left.2\left(q_{0}^{2}+\delta_{x x}^{2}\right) \delta_{x} a_{1}\right) /\left(\delta_{x} A_{0}, \delta_{x} A_{0}\right)
\end{aligned}
$$

and

$D_{\perp}=2\left(\partial_{x} A_{0},\left(q_{0}^{2}+\partial_{x x}^{2}\right) \partial_{x} A_{0}\right) /\left(\partial_{x} A_{0}, \partial_{x} A_{0}\right)$.

Close to the threshold, using expansion (4) for $A_{0}$ the diffusion coefficients read [9] :

$$
\begin{aligned}
& D_{\|}=4 q_{0}^{2} \frac{\varepsilon-12 q_{0}^{2} \delta^{2}}{\varepsilon-4 q_{0}^{2} \delta^{2}}, \\
& D_{\perp}=4 q_{0}^{2}\left[\frac{\delta}{q_{0}}+\frac{\left(\varepsilon-4 q_{0}^{2} \delta^{2}\right)^{2}}{1024 q_{0}^{8}}\right] .
\end{aligned}
$$

A negative diffusion coefficient is associated with an instability of the roll structure [10]. In the plane $(\varepsilon, \delta)$ the curve $D_{\|}=0$ delineates the region where the convective state is unstable against a longitudinal compression of the structure. As already found by Eckhaus [11] this occurs when $q$ lies in one of the two ranges

$\left(q_{0}-\delta_{\|}, q_{0}-\delta_{\|} / \sqrt{3}\right)$ and $\left(q_{0}+\delta_{\|} / \sqrt{3}, q_{0}+\delta_{\|}\right)$.

The rolls are also unstable against torsion when $D_{\perp}<0$, that is to say when $q<q_{0}+\delta_{\perp}(\varepsilon)$, where $\delta_{\perp}(\varepsilon)$ is given by eq. (5). This may lead to an explanation for the low frequency turbulence observed by Ahlers and Behringer [12] close to the onset of convection in wide cells. Of course, the gradient flow defined by eq. (2) cannot lead to a permanent unsteady state since in a finite box $V[A]$ reaches asymptotically in time its lower bound which corresponds to a stationary solution, however this steady state is marginally unstable against torsion $[q$ being given by (5) implies $\left.D_{\perp}=0\right]$. But eq. (2) describes the actual situation only imperfectly. In the R.B. case other non-linear terms such as $(\nabla A)^{2} A$ are expected on the r.h.s. of (2), destroying the gradient flow property. Numerical experiments with this term added show that the wave-length increase still remains and we conjecture that this brings the structure into an unstable state with $D_{\perp}<0$ which behaves in an erratic way instead of having a well defined structure as it would be the case if there were still a Liapounov functional.

3. Thermal phase fluctuations. - In the steady state, thermal fluctuations are described by the probability density functional

$$
\mathfrak{T}[A] \propto \exp \left\{-\mathcal{U}[A] / k_{\mathrm{B}} T\right\}
$$

where the thermodynamic potential $[3 a] \cup[A]$ is the product of our dimensionless potential $V A$ ] given by (4) times a typical energy $\mathcal{E}$ (per unit surface) stored in the structure. $k_{\mathrm{B}} T$ is the thermal energy at temperature $T$. In all realistic cases $k_{\mathrm{B}} T$ is so small that fluctuation effects seem difficult to observe. However the case of phase fluctuations seems more favourable than that of amplitude fluctuations. To estimate this effect we need the potential variation $\delta^{2} V[\varphi]$ for a phase fluctuation of the system of parallel rolls :

$$
\begin{aligned}
\delta^{2} V[\varphi]=\frac{1}{2} \int \mathrm{d} x \mathrm{~d} y\left(\partial_{x} A_{0}\right. & \left., \partial_{x} A_{0}\right) \times \\
& \times\left[D_{\|}\left(\partial_{x} \varphi\right)^{2}+D_{\perp}\left(\partial_{y} \varphi\right)^{2}\right] .
\end{aligned}
$$

[From this, one gets back eq. (8) using the gradient flow property.] The probability distribution for a Fourier component of a longitudinal phase fluctuation reads $T\left(\varphi_{q}\right) \propto \exp \left\{-\left|\varphi_{q}\right|^{2} / 2\left\langle\left|\varphi_{q}\right|^{2}\right\rangle\right\}$ where, from the Equipartition theorem :

$$
\left\langle\left|\varphi_{q}\right|^{2}\right\rangle=k_{\mathrm{B}} T / E \times\left(\partial_{x} A_{0}, \partial_{x} A_{0}\right) \times D_{\|} q^{2} .
$$

$E=\varepsilon L_{x} L_{y}$ is the total convective energy far from the threshold (typically $\varepsilon=1$ ), $L_{x}, L_{y}$ being the horizontal dimensions of the convective layer. From expansion (4) for $A_{0}$ one gets : $\left(\partial_{x} A_{0}, \partial_{x} A_{0}\right)=\frac{2}{3} \cdot q_{0}^{2} \varepsilon$, so that $\left\langle\left|\varphi_{q}\right|^{2}\right\rangle$ diverges as $\varepsilon^{-1}$. The dynamics of the fluctuation $\varphi_{q}$ obey the Langevin equation

$$
\partial_{\mathrm{t}} \varphi_{q}=-D_{\|} q^{2} \varphi_{q}+f_{q}(t)
$$

where $f_{q}(t)$ is a gaussian white noise such that $\left\langle f_{q}(t)\right\rangle=0$ and $\left\langle f_{q}(t) f_{-q}\left(t^{\prime}\right)\right\rangle=F_{q} \delta\left(t^{\prime}-t\right)$. From the Fluctuation-Dissipation theorem $F_{q}$ is equal to $2 q^{2} D_{\|}\left\langle\left|\varphi_{q}\right|^{2}\right\rangle=3 k_{\mathrm{B}} T / E q_{0}^{2} \varepsilon=F$, independent of $q$. The $(q=0)-$ Fourier component just defines the position $X$ of the roll system taken as a whole. Due to phase fluctuations, $X$ exhibits a brownian motion ; from a straightforward integration of (10) 
with $q=0$ one gets : $\left\langle(X(t)-X(o))^{2}\right\rangle=2 \bar{D} t$ with $\bar{D}=F / 2$. As this effect is connected with translational invariance, one has to consider a geometry where the position of the rolls is not controlled by the lateral boundaries parallel to the roll axis ( $y$ direction). This is the case of the Stork and Muller experiment [13] : a horizontal annular cell of height $d$, mean perimeter $L_{x}$ and width $L_{y}$ where the roll pattern assumes a radial disposition [In order that the compression-type movement be the only possible the ratio of the length of the roll to their diameter $\left(\sim L_{y} / d\right)$ should be small enough]. Let

$$
\bar{X}=\frac{q_{0}}{\pi}\left(\left\langle(X(t)-X(o))^{2}\right\rangle\right)^{1 / 2}
$$

be the mean displacement (in units of the roll diameter) one gets :

$$
\bar{X}^{2}(t)=\frac{3 k_{\mathrm{B}} T}{\pi^{2} E \varepsilon} \times \frac{t}{t_{0}}
$$

where $t_{0}$ is a characteristic time relevant to the problem. For R.B. instability in liquid helium in the conditions of ref. [12] at $T \cong 5 \mathrm{~K}$ with $d \simeq 0.2 \mathrm{~mm}$, $L_{y} \simeq 5 d, L_{x} \simeq 1 \mathrm{~cm}$, for $\bar{X}=0.1$ and $\varepsilon=10^{-2}$ one gets $t_{\mathrm{B}}=6 \times 10^{4} \mathrm{~s}$. For the Carr-Helfrich instability in nematics [14], orientational elasticity plays the dominant role ; $\mathcal{K} \sim K / d$ is the Frank elastic energy per unit surface and $t_{0}=\gamma d^{2} / K$ is the orientational relaxation time. Assuming $d \sim 10 \mu \mathrm{m}$, $L_{y} \sim 1 \mathrm{~mm}, L_{x} \sim 1 \mathrm{~cm}$ and $T \sim 300 \mathrm{~K}$ leads to $t_{\mathrm{B}}=8 \times 10^{4} \mathrm{~s}$ for $\bar{X}=0.1$ and $\varepsilon=10^{-2}$.

Repeating the argument at a more qualitative level for a small portion of the structure (of length $l_{x} \sim$ a few roll diameters) one gets a much smaller brownian diffusion time $t_{\mathrm{B}} \sim l_{x}$; now the whole structure rearranges itself in a time $t_{\mathrm{R}} \sim L_{x}^{2}$ and a brownian local disturbance can be observed if the cell is long enough so that $t_{\mathrm{R}} \gg t_{\mathrm{B}}\left(\bar{X}_{10 \mathrm{c}}\right)$. Experiments could be performed in helium using local probes [15] or in nematics by direct microscopic observation.

\section{References}

[1] Normand, C., Pomeau, Y., Velarde, M., Rev. Mod. Phys. 49 (1977) 581

[2] Koschmieder, E. L., Pallas, S. G., Int. J. Heat Mass Transfer 17 (1974) 991 ;

Bergé, P., Dubois, M., Phys. Rev. Lett. 32 (1974).

[3a] Graham, R., in Fluctuations Instabilities and Phase Transitions (T. Riste ed.). NATO Adv. Study Inst. Vol. B11 (Plenum Press, New York) 1975.

[3b] Zaitsev, V. M., Shliomis, M. I., Sov. Phys. J.E.T.P. 32 (1971) 866.

[4] Landau, L. D., Dokl. Akad. Nauk. SSSR 44 (1944) 339.

[5] Swift, J., Hohenderg, P. C., Phys. Rev. A 15 (1977) 319.

Strictly speaking, the linear part of the r.h.s. of (2) has been derived for perturbations with wavenumber $q \sim q_{0}$ only. However we assume that it describes also the linear damping of fluctuations with any wavenumber. This could be relaxed by replacing this linear operator by a more general symmetric integral kernel of the form $\int \mathrm{d} x^{\prime} \mathrm{d} y^{\prime} K\left(\left|\mathbf{r}-\mathbf{r}^{\prime}\right|\right) A\left(\mathbf{r}^{\prime}\right), \quad \mathbf{r}=(x, y)$. This does not change at all one of our main results : the preferred wavenumber is on the curve $D_{\perp}(\varepsilon, q)=0$. This last result remains valid too if the non linear term on the r.h.s. of (2) is, instead of $A^{3}$, of the general form $\frac{\mathrm{d} U(A)}{\mathrm{d} A}$, where $U$ is an even function such that $U(A) \simeq \frac{A^{4}}{4}+O\left(A^{6}\right)$ and $\frac{\mathrm{d}^{2} U(A)}{\mathrm{d} A^{2}}>0$. Moreover the wavelength increases with $\varepsilon$ whatever the linear term in (2) is, provided that the linear damping rate increases with the wavenumber for $q>q_{0}$, which is realized by most realistic systems.

[6] With boundary conditions $A=\nabla A=0$ one recovers from (2) a boundary layer of thickness of order $\varepsilon^{-1 / 2}$ perpendicular to the roll axis and $\varepsilon^{-1 / 4}$ parallel to this axis, near $\varepsilon=0_{+}$ (Brown, S. N. and Sterwardson, K., Studies in Appl. Math. 57 (1977) 187; Segel, L. A., J. Fluid Mech. 38 (1969) 203).

[7] A good account of the Chapman-Enskog theory is in Chap. 13 of Equilibrium Statistical Mechanics by BaLesCU, R. (Wiley Pub., New York) 1975.

[8] This slow diffusion of roll systems has been analyzed near the threshold in Newell, A. C. and WhiteheAD, J. A., J. Fluid Mech. 38 (1969) 279.
[9] Using results of WeSFreid et al. (J. Physique (Paris) 39 (1978) 725 ) one can derive the physical expressions of the so far dimensionless results for the R.B. case with the realistic rigid-rigid boundaries. One gets

$$
\begin{aligned}
& D_{\|}=Q \chi\left(\tilde{\varepsilon}-12 \frac{\delta^{2}}{q_{0}^{2}}\right) /\left(\tilde{\varepsilon}-4 \frac{\delta^{2}}{q_{0}^{2}}\right), \\
& D_{\perp}=Q \chi\left\{\frac{\delta}{q_{0}}+\frac{\left[\tilde{\varepsilon}-4\left(\delta^{2} / q_{0}^{2}\right)\right]^{2}}{1024}\right\}
\end{aligned}
$$

where $\chi$ is the thermometric diffusivity,

$$
Q=5.69 P /(1+1.95 P),
$$

$P$ the Prandtl number ; the critical wave vector $q_{0}$ is the $3.117 d^{-1}, d$ being the thickness of the layer and

$$
\tilde{\varepsilon}=2.782 \varepsilon=2.782\left(R-R_{\mathrm{c}}\right) / R_{\mathrm{c}} .
$$

[10] Other kinds of instabilities exist within our formulation. For example the oblique-roll instability (of which the cross-roll instability is particular case) is found when considering perturbations with a wave vector $q_{0}+\delta$ making a finite angle with the wave vector $q_{0}+\delta_{0}$ of the underlying convective structure. In our case the corresponding stability region is defined by $\varepsilon \geqslant 8 q_{0}^{2} \delta_{0}^{2}$ so that this oblique-roll instability is less dangerous than the Eckhaus instability (E.I.). However contrary to the case of the E.I. the boundary of the cross-roll instability is not universal since it is a function of the Prandtl number, of the type of boundary conditions and depends also on the details of the angular dependence of the interaction between different wave-vectors, all things which are clearly not contained in our simplified description.

[11] EckHAus, W., Studies in Non-Linear Stability Theory (SpringerVerlag, New York) 1965

[12] Ahlers, G., Behringer, R. P., Phys. Rev. Lett. 40 (1978) 712.

[13] Stork, K., Muller, V., J. Fluid Mech. 71 (1975) 231.

[14] Goossens, W. J. A., in Adv. Liq. Cryst. Vol. 3, Brown ed. (Academic Press, New York) 1978.

[15] LibChaber, A., MaURer, J., J. Physique Lett. 39 (1978) L-369. 